\title{
Designing Autonomy in Cars: A Survey and Two Focus Groups on Driving Habits of an Inclusive User Group, and Group Attitudes Towards Autonomous Cars
}

\author{
Ioannis Politis ${ }^{1}$, Patrick Langdon ${ }^{1}$, Mike Bradley ${ }^{1}$, \\ Lee Skrypchuk ${ }^{1,2}$, Alexander Mouzakitis ${ }^{2}$, and P. John Clarkson ${ }^{1}$ \\ ${ }^{1}$ Engineering Design Centre, Department of Engineering, University of Cambridge, \\ Trumpington Street, Cambridge, CB2 1PZ, UK \\ \{ip332, pml24, mdb54, 1s578, pjc10\}@cam.ac.uk \\ ${ }^{2}$ Jaguar Land Rover, Research \& Technology, Coventry, UK \\ $\{$ LSkrypch, AMouzak1\}@jaguarlandrover.com
}

\begin{abstract}
Autonomous driving is a topic of extensive research; however user views on this new technology are largely unexplored, especially for an inclusive population. This paper presents a survey and two focus groups, investigating driving habits and attitudes towards autonomous cars of an inclusive group of UK drivers. A subset of survey participants were invited to attend one of two focus groups, to discuss handovers of control between car and driver. Maintaining safety, trust and control were themes commonly identified in both focus groups, while unique views and concerns, relating to different characteristics of the group were expressed. These results can inform an inclusive, user-centred design of autonomous vehicle interfaces, especially for the safety-critical use case of driver handovers of control.
\end{abstract}

Keywords: Human Factors · Autonomous cars · Survey · Focus group Handover of control

\section{$1 \quad$ Introduction}

Autonomy in cars is becoming a reality, with an ever-increasing number of manufacturers predicting widespread availability of autonomous driving solutions in the next five years [1-3]. The public's expectation is that this technology will deeply affect the norms of transportation, and the dynamics of car ownership [4]. In the midst of this significant transition, potential users of autonomous cars have been given the opportunity to express their views, hopes, and concerns for a future where self-driving cars will be widely available, e.g. [5-10]. However, two critical considerations are lacking; firstly, available work related to how current driving habits correlate with the perceived need for autonomy is sparse, especially in the UK [11]. Secondly, 
consideration of the population as a set of users with varying degrees of capabilities to be accommodated, as described in inclusive design [12], has been rare [13].

This paper begins to address this problem, by investigating driving habits and opinions of a set of users in the UK, through an online survey conducted between July and November 2016. The survey, along with general questions on demographics and driving habits, investigated self-reported identification with UK driver profiles, as identified by a UK-wide capability study conducted in 2015 by Transport Systems Catapult [14]. It also provided a set of questions regarding self-reported capabilities, as used in an inclusive design methodology [15], never before used in the context of autonomous cars. The relation of the above factors to current driving enjoyment and acceptance of the idea of autonomous cars provided an interesting set of insights on the views of drivers about autonomy.

A subset of the survey respondents were invited to attend one of two focus groups at the University of Cambridge, to share ideas specific to the problem of handover of control between, car and driver, which is expected to be of particular importance in autonomous cars Human-Machine Interface (HMI) design [16, 17]. During a handover, vehicle control is transferred between the car and the driver, for example due to driver choice or limitations of automation. Focus group participants were presented with the problem and asked to share their views during an interactive session with the assistance of visual and tangible aids. In accordance with an inclusive design approach, participants of distinct characteristics regarding age, gender, and technological expertise were invited in each focus group, providing an initial but extensive set of views on autonomous cars and handovers. Further, interesting commonalities were found, but also unique views expressed amongst participants of the two focus groups.

\section{$2 \quad$ Related Work}

\subsection{User views}

As car autonomy is entering the mainstream in driving technology, recent studies have investigated user views on autonomous cars. KPMG LLP [18] reported a focus group conducted with US drivers in 2013. Participants expressed concerns on safety, liability, and vehicle handling, but they were positive towards reduced expected commute times, and avoidance of traffic. They were expecting to be able to switch automation on and off when using an autonomous car. Rödel et al. [5] reported a survey on levels of autonomy with members of the University of Salzburg, conducted in 2014. It was found that perceived control and fun decreased as the degree of automation increased ${ }^{1}$. User acceptance and perceived user experience was higher with cars similar to the ones on the roads at the time, but pre-existing experience with Advanced Driver Assistance Systems (ADAS) positively affected these metrics for autonomous vehicles.

Kyriakidis, Happee \& de Winter [10] presented in 2015 a large scale survey on public opinion on automated driving from 109 countries. It was found that manual

\footnotetext{
${ }^{1}$ The levels of autonomy considered in that study were published by the National Highway Traffic Safety Administration (NHTSA) in 2013 [31]. NHTSA has since started using the autonomy levels published in SAE J3016 standard [32].
} 
driving was the most enjoyable mode of transportation. Further, there were split opinions on how much respondents were willing to pay for an autonomous driving function (ranging from zero to tens of thousands of dollars). Concerns were expressed about security, liability and safety of autonomous cars. Bazilinskyy, Kyriakidis \& de Winter [19] processed the free text responses of [10] in a crowdsourcing study presented in 2015, revealing split opinions between positive and negative comments on automated driving, and further highlighting this polarisation of views.

Schoettle and Sivak $[6,7]$ considered the public opinion on autonomous vehicles from users in China, India, Japan, US, UK and Australia, in two 2014 surveys. There were high expectations on benefits of autonomous cars, but high level of concerns regarding riding on these vehicles, especially in terms of security, possible system failures, and performance. The majority of respondents would not pay extra in order to have autonomous vehicles (except users in China and India). Regarding drivers' preferences on vehicle automation, Schoettle and Sivak $[8,9]$ presented results of two surveys conducted in 2015 and 2016 with US drivers. Results were consistent in both surveys, and showed that no self-driving capability was the most preferred, with partially self-driving coming next, and fully self-driving being the least preferred mode. In a fully self-driving mode the highest concerns were expressed, while a desire to have the option to take over, and to be notified multimodally on this event was expressed.

To conclude, although excitement is high, the public worldwide has expressed reservations regarding autonomy in vehicles, especially in terms of safety and liability. Retaining the option of control seems to be of vital importance for perceived user experience. Studies with focus in the UK are lacking, with a 2014 survey by Ipsos MORI being one of the few available [20]. In that work, there was limited perception of importance of driverless technology, and perceived usefulness was higher with Londoners. Older respondents (aged over 55) were less willing to use this technology, while non-driving enthusiasts were more likely to accept this technology. This study, while useful, did not explicitly address the capabilities defined in inclusive design, while interaction with users was only in the context of a survey, with no follow-up discussions. Elaborating on the views of an inclusive user group in the UK is therefore essential, and would aid a design for a wider range of capabilities.

\subsection{Inclusiveness}

Inclusive design [12] considers a diverse user group in terms of capabilities in the design cycle. Viewing the population as either being "able-bodied" or "disabled" can be limiting when designing for users who would not identify themselves with any of the above groups. Difficulties can be present in everyday activities, ranging from hearing or vision, memory or thinking, communication, mobility and dexterity, without necessarily leading to a person self-identify, or to have sufficient capability loss, to be classified as having a disability. A lack of technological prior experience can also lead to exclusion from being able to operate technologies with complex interfaces, for example by those who are considered digitally excluded [21-23]. Inclusive design aims to address these varying characteristics to create complete and usable solutions, rather than design specialist products. By detecting difficulties when using a design and considering how these affect the usability of the product, it aims to provide solutions 
that can satisfy a wider range of the population. In the field of autonomy in vehicles, this methodology, while promising, has not been widely applied [13].

Körber \& Bengler [24] provide a review of potential individual factors that could influence interaction with automation. As such factors, they identify Dispositional Factors (relating to performance), Traits (relating to task engagement), Driver State (relating to fatigue), Attitudes (relating to trust in automation), and Demographics and Other Factors (relating to age, experience with ADAS, behavioural disorders, and working memory). Although broad, this classification is not as heavily focused on the individual and less transient characteristics of the users, which under Körber \& Bengler's categorisation would possibly belong to Demographics and Other Factors category. Souders \& Charness [25] focus on age as a defining characteristic of technology acceptance for autonomous vehicles, and identify trust and familiarity in the new technology as decisive factors for adoption by older adults. To this end, along with demographics and driving habits, an assessment of self-reported performance in vision, hearing, managing tasks of daily life, mobility or physical movement, and tasks that require precise hand movements was followed, in order to create a complete picture of user capabilities in everyday activities, as suggested in inclusive design.

The present study begins to address the exciting opportunity to utilize an inclusive design thinking as early as possible in the development of autonomous car technology. Other than Kunur et al. [26], who studied future car concepts with no explicit focus on autonomy, no study has utilised this methodology in the past in the context of autonomous vehicles. Doing so, can increase focus on user needs in the resulting concepts, identifying the population as an inclusive one from early on in the design process. Combining two different tools to detect user views, i.e. a survey and a focus group, can elicit distinct findings, which together can inform the creation of safe and inclusive HMI concepts for autonomy. The remainder of this paper will summarise the survey used as an initial tool for identifying characteristics of a small user population, and as a recruitment tool for a series of focus groups to follow. Two focus groups with a subset of survey participants will then be described, which helped to identify different user views on the topic of autonomous cars and handovers of control.

\section{Survey}

\subsection{Description}

A survey comprising questions on demographics, driving habits, and a self-reported assessment of capabilities was administered, using social networks, online mailing lists and paper advertisements distributed in the city of Cambridge ${ }^{2}$. After asking whether participants had a driving licence, their most regular forms of transport were asked (car, public transport, bicycle etc.), as well as the type of journeys most frequently made with a car (rural, suburban or urban). The frequency with which respondents drove (daily, weekly, monthly etc.), and their estimation on annual mileage were then asked. The survey continued with asking participants to self-categorise into one of the traveller profiles identified in Traveller Needs and UK Capability study by Transport Systems

\footnotetext{
${ }^{2}$ The survey used Google Forms and can be found in https://goo.gl/Ya88ds .
} 
Catapult [14]. An effort was made to reflect the characteristics of the profiles in [14] into a set a set of items that would be easy for the survey participants to respond to. The description of profiles in [14] and the respective questions in the present survey can be found in Table 1. The survey continued with asking about how much the respondents enjoyed driving, and how much they liked the idea of autonomous cars. Both questions used 5-point Likert scale items, and were used as simple measures of user acceptance for both manual and autonomous driving. Since autonomous cars are not prevalent on the roads yet, the appeal of the idea of these cars was asked instead of current enjoyment. The survey continued with a set of questions on self-reported capabilities with vision, hearing, managing tasks of daily life (assessing thinking and communication), mobility or physical movement (assessing reach and stretch), and with tasks requiring precise hand movements (assessing dexterity). The questions were used as a short self-assessment of capabilities, and were modified from [15] and used information from the Inclusive Design Toolkit [27]. All questions were using a 4-point Likert scale, in line with [15], and optional comments on any difficulties were asked by participants. Finally, the respondents' age, gender, and country of residence were asked, as well as their intention to participate to a following focus group.

Table 1: The profiles identified in Traveller Needs and UK Capability study [14] (A), and their interpretation in survey items in the present study (B).

\section{A: Traveller Types}

Progressive Metropolites: Living in the heart of the city, typified by the technology-savvy young professional, with significant amounts of personal and business travel. Want to reduce their transport footprint.

Default Motorists: High mileage drivers, with a mix of those who enjoy driving and many for whom it is a functional choice.

Dependent Passengers: Dependent on others for their mobility needs, representing a mix of students, elderly, and those with impairments.

Urban Riders: City dwellers, who travel less frequently than the Progressive Metropolites, making use of public transport available to them.

Local Drivers: Mainly retirees or stay at home parents, making low mileage local journeys.
B: For what reasons do you typically drive a car?

Choose one or more.

$\square \quad$ As part of my job

$\square \quad$ For commuting to work

$\square \quad$ For everyday small trips

$\square \quad$ For taking children to school and household needs

$\square \quad$ For the enjoyment of driving

$\square \quad$ Because I have no other way of doing my journeys

$\square \quad$ I don't typically drive, I am usually a passenger

$\square \quad$ Other

\subsection{Results}

In total there were 97 respondents to the survey. Out of these, 63 were living in the UK and had a driving licence, for which results are reported in this paper. There were 25 female respondents, 37 male, and one preferred not to say. Descriptive statistics of the responses are presented in Table 2 . 
Table 2: Descriptive statistics of the survey on driving habits. Note that some of the below categories (indicated by *) are not mutually exclusive. In these categories, one person can have multiple responses, for example, they may both use car and public transport as means of transport. In all cases, absolute numbers are reported.

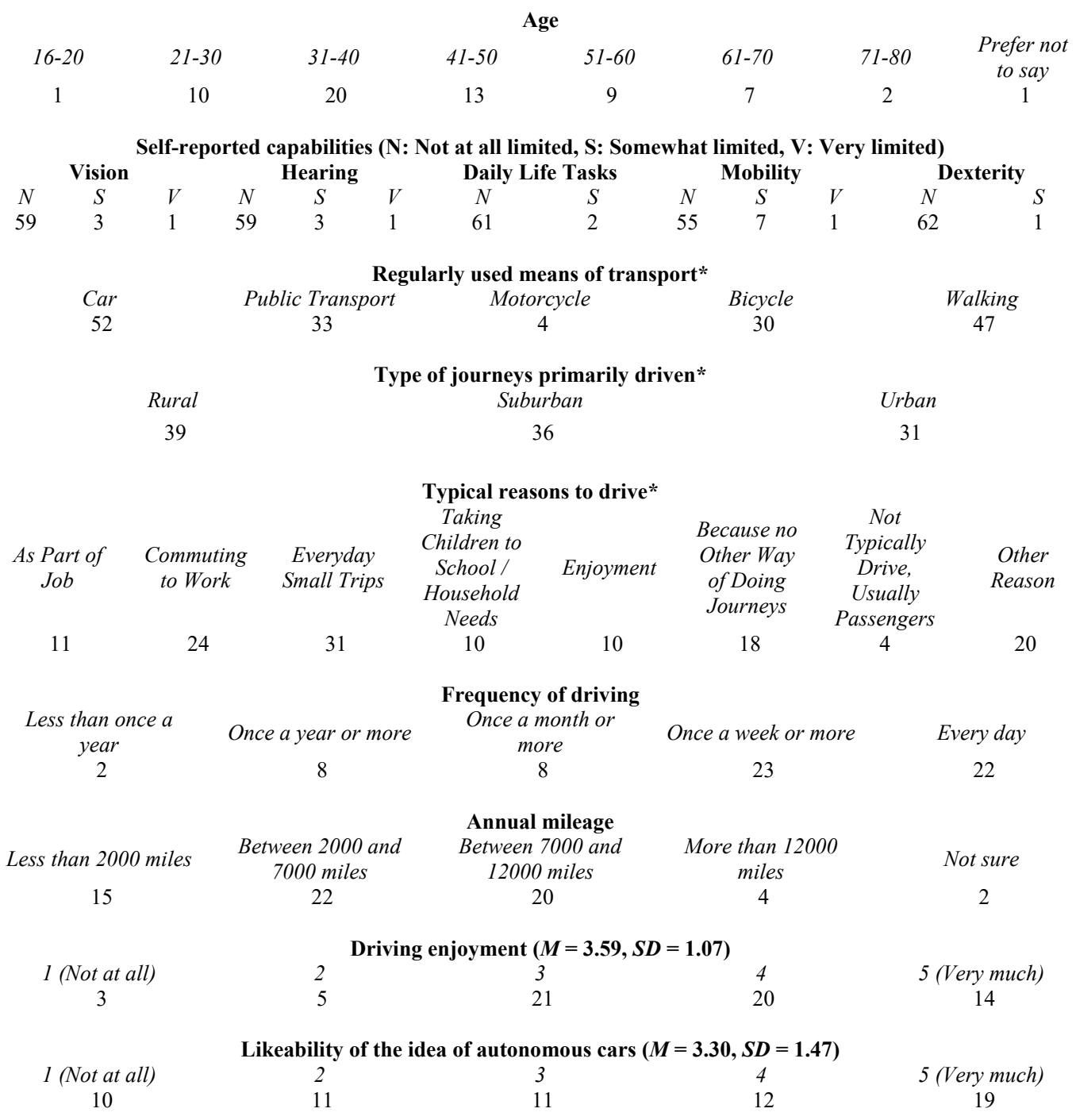

Independent samples t-tests showed that respondents who used a car as part of their job enjoyed driving more compared to respondents who did not $(t(61)=-2.78, p<0.01)$. Further, respondents who used a car for everyday small trips enjoyed driving more compared to respondents who did not $(t(61)=-3.23, p<0.01)$. As expected, respondents who used a car for the enjoyment of driving enjoyed driving more 
compared to respondents who did not $(t(26.94)=-4.37, p<0.001)$. Finally, respondents who used a car for other reasons to the ones mentioned in the survey enjoyed driving less compared to participants who did not $(t(61)=2.60, p<0.05)$. Other reasons for driving mentioned were mostly related to holidays and social visits.

A one-way ANOVA with frequency of driving as a factor revealed a significant main effect to driving enjoyment $(F(4,58)=2.92, p<0.05)$. Planned contrasts revealed that respondents who drove less than once a year enjoyed driving less compared to respondents who drove once a year or more $(t(58)=-2.35, p<0.05)$, once a month or more $(t(58)=-2.20, p<0.05)$, once a week or more $(t(58)=-2.95, p<0.01)$ and every day $(t(58)=-3.16, p<0.01)$. No other significant effects were found regarding how driving habits and user characteristics affected driving enjoyment, and no significant effects were found regarding how driving habits and user characteristics affected likeability of the idea of autonomous cars. Regarding inclusive user characteristics of the specific group of respondents, there were limited responses indicating any difficulty in everyday life (see Table 2).

The respondents of the survey were low in number, limiting the generalisability of the results presented. Future work can distribute this survey in a wider UK population, in order to present more extensive results, using a more inclusive set of users in terms of self-reported capabilities. However, as discussed, this survey was used mainly as a tool to recruit participants for the focus groups, and as such it achieved its goal. Further, the characteristics of the population presented are not dissimilar with the ones presented by larger scale surveys reviewed in Section 2.1. Finally, the statistically significant results observed provide confidence that a larger population can display even richer effects. A subset of survey respondents were invited to one of two focus groups at the University of Cambridge, to be described in the next section.

\section{Focus Groups}

\subsection{Description}

In order to elicit more elaborate discussions on the topic of autonomous cars, two focus groups with participants of the survey were conducted at the University of Cambridge in July and August 2016, Focus Group 1 (FG1) and Focus Group 2 (FG2). The topic of interest for both was handovers of control in autonomous cars. As discussed earlier, handovers are situations where the car transfers control to the driver or vice versa. These can occur for various reasons and can be voluntary or otherwise. This topic was selected as one of particular interest to the industry, as well as one that is expected to become especially relevant as cars become more autonomous [28]. Previous studies have explored the interactions that can occur in such a situation, for example by investigating appropriate warnings to deliver to drivers [29]. However, exploring participants' thoughts on handovers in the context of a focus group has not been previously attempted. In order to invite the thoughts of sufficiently different user groups, the focus groups used different demographics.

FG1 consisted of 5 males aged between $31-39$ years $(M=35.00, S D=4.00)$, had driving experience between 11 and 19 years $(M=15.40, \mathrm{~S} D=3.05)$, and practiced mostly technical professions (Project Architect, Hardware Design Engineer, Motion 

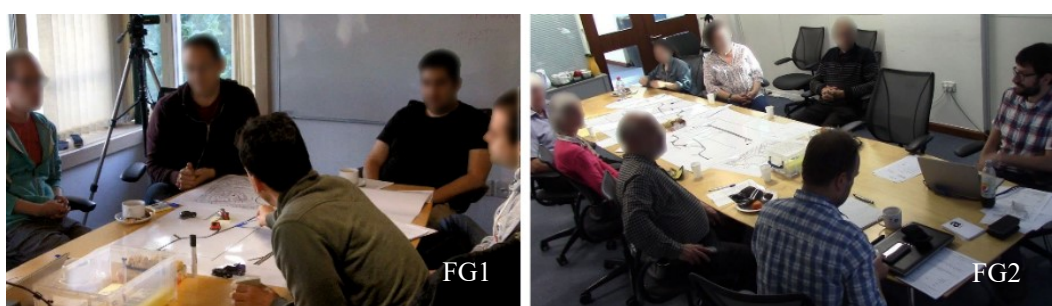

Fig. 1. The setup of to the two focus groups (FG1 on the left and FG2 on the right)

Graphics Designer, Research Engineer, Quality Control Assistant). FG2 consisted of 3 females and 3 males, aged between $24-79$ years $(M=55.83, S D=18.93)$, had driving experience between 5 and 62 years (one participant did not report years of driving experience, for the rest: $M=34.00, S D=21.14)$, and practiced a mixed set of professions (one person did not report profession, for the rest: Retired Government Officer, Designer, Retired Motor Engineer, Illustrator, Charity Worker).

Both focus groups had identical format. Aside to the focus group participants, two researchers were facilitating the discussions and taking notes. The focus groups were recorded and filmed with the participants' consent, and a computer presentation, corresponding to all parts of the focus group, was always projected. Further, there were schematics of different road types (a highway, a city road, and a roundabout) available as large prints, as well as miniature cars, to be used in any part of the discussion. In the beginning, participants signed consent forms, and received web stores vouchers for their time, while refreshments and light food were available. They were informed that there were no right or wrong answers in the discussions, since their views were sought, and that any data received would be anonymised. After the initial introductions of participants and researchers, the topic of handovers was introduced, using schematics and videos through the presentation. Any initial thoughts on the topic were discussed, using unstructured discussions. This led to the next part of the focus group, where two specific scenarios requiring handovers were discussed. These were focusing on the case where a driver would need to take control from the car, either before an exit on the highway, or before a roundabout in the city. Any additional thoughts about each of the two scenarios were discussed, with focus in how the car should be like, and how it should behave, aiming to elicit comments on the car's HMI design. For these two scenarios, aside to the unstructured discussions, a set of questions were always visible to the focus group participants in the presentation, to assist more focused discussions. These were related to what would happen in these scenarios, and when and how would it happen, what would the driver need to know, who would be involved, and any other thoughts. After asking for any final thoughts or comments, the focus groups were concluded. They lasted about two hours each. See Figure 1 for the setup used.

\subsection{Results}

The results of the focus were analysed thematically by three coders, which were members of the research team, as suggested in [30]. $\mathrm{NVivo}^{3}$ software was used to create

\footnotetext{
${ }^{3} \mathrm{http}: / /$ www.qsrinternational.com/
} 
thematic nodes, and each coder's analysis was iterated and revisited by the next coder. This resulted in two thematic analyses, one for each focus group. The main views discovered can summarised on Table 3 .

As can be seen on Table 3, the views discovered in the two focus groups bear similarities. A need for the driver to be informed during the process of handovers was expressed by both groups, proposing multimodal displays (FG1) or saliency in general (FG2) as solutions. Further, simplicity of the HMI in autonomous cars was desired by both groups. An improved safety was expected and required by both groups, when autonomous driving technology becomes mainstream. A point made by participants of both focus groups was that autonomy is useful only if it is complete, due to potential complexities in driving partially autonomous cars. This view received no clear consensus in neither of the groups, but it was a seemingly strong one for the participants who shared it. However, notable differences between the focus group views were also observed. Participants of FG1 put emphasis in retaining control of the handover situation, having the final say on who will have control. This seemed to be less the case for FG2 participants, who were mostly concerned about whether vehicle and HMI technologies will be mature enough to accommodate an autonomous functionality, and whether it will be safe and robust enough. Conversely, FG1 participants expressed concerns related to hard ethical questions on liability in case of accidents with an autonomous car, as well as concerns related to loss of jobs and exploitation of privacy. FG1 participants, however, also saw an opportunity for a better use of the driver's time in autonomous cars. They felt that autonomous cars can assist them in becoming even better and more efficient in their activities. In contrast, FG2 participants required autonomy to address limitations of the driver's capabilities, such as sight problems, adjust to their driving style, and facilitate driver comfort through easy-to-use HMIs, such as voice-based ones. They also required a comfortable period to signify a handover to the driver, aided by communications between cars and infrastructure.

Table 3: A summary of the views discovered in the focus groups, sorted by the frequency of occurrence of the respective themes in the thematic analysis.

\section{Focus Group 1}

- Multimodal displays that maintain the driver's situation awareness during handovers are required, to aid safety.

- HMIs designed for handovers should be simple and easy to use.

- The driver should be the one who decides whether to give or take control of driving.

- Cars should either have full autonomy or no autonomy.

- Self-driving cars can provide a better use of the driver's time, safety and efficiency.

- Self-driving cars can create ethical questions in case of incidents, loss of jobs, and privacyrelated concerns.

- Handovers should be safe.

- Autonomous cars should enhance the driver's capabilities.

\section{Focus Group 2}

- There is concern whether autonomous cars will be able to cope with difficult road scenarios, and whether their interfaces will be robust enough.

- Autonomous cars would need to address limitations in everyday life activities, such as sight problems, by simplifying the HMI, using for example voice control.

- Vehicle to vehicle and vehicle to specialized infrastructure communications can create safer future roads.

- Handovers should be communicated saliently by the HMI well before they need to happen.

- Autonomous cars are expected to increase road safety and comfort.

- Cars should either have full autonomy or no autonomy.

- Autonomous cars should adjust to the driver's driving style. 


\section{Discussion}

The results of the survey, although with a small sample of respondents, showed a good distribution of group characteristics. There was representation of all age ranges and coverage of all driving profiles. Since this survey is the first to the authors' knowledge combining questions on inclusive characteristics, driving habits, driving enjoyment, and likeability of the idea of autonomous cars, it is difficult to make direct comparisons with available literature in terms of responses. It is argued, however, that the combination of these questions covered a wide range of information, and can be used as tool to recruit a more inclusive participant sample for later studies, such as focus groups (as done successfully in this paper) and experiments (as planned for future work). It is also expected, that a larger sample of respondents will reveal even clearer patterns on how driver characteristics correlate with driver attitudes towards manual and autonomous cars. However, even using this small sample of respondents, some clear patterns emerged. These related to driving enjoyment, and showed that participants who drove more or drove due to their work enjoyed driving more. No such pattern was found for the likability of the idea of autonomous cars. This is comparable to the results of $[5,8-10]$, where manual modes of driving were considered as the most enjoyable and accepted. There still seems to be road to cover in terms of improving acceptance of autonomous cars, as also found for the UK in [20], and this study showed no evidence to the contrary. In the authors' view, and as also posited by [5, 25], a more widespread exposure to the technology may mitigate concerns, and improve trust. Engaging an inclusive user group during the design process is a step in this direction.

The results of the focus groups showed clear evidence on why an inclusive design process can be beneficial. This is because similar views on major topics of concern were discovered, but also distinct points were made by the groups in areas of interest for inclusive design. Participants of both focus groups were interested in a technology that will be safe and will assist their everyday needs, a popular finding also in studies like $[6,7,10,18]$. However, FG1 participants saw the technology as augmenting their already high everyday capabilities, and allowing them to be productive in autonomous mode, with them still having the final say in things. Being a highly technical group, they were mostly worried about social implications of autonomy, and less about robustness. FG2 participants on the other hand expected the technology to be robust, to not fail, to be comfortable, and to account for possible own limitations in everyday life. This is a new result, and highlights the different views of this technology for groups of different capabilities. It can help autonomous vehicle designers increase their inclusivity, by providing solutions a wider spectrum of drivers can benefit from. On the topic of in-car displays, the need for clear, easy to interpret, multimodal information during handovers was a point of consensus between the two groups. Available literature (e.g. [29]) has looked into the effectiveness of such warning mechanisms, but never before has this requirement been confirmed in the context of a focus groups with such varying demographics. Autonomous vehicle designers can benefit from this guideline, by creating usable and inclusive interfaces with salient cues, that are easy to react to. Finally, the polarisation of views on whether partial autonomy is acceptable, again by both groups, raises an issue of acceptance. Some participants were concerned that partial autonomy might be more of a burden than a liberator, due to high demand from the driver. This point needs to be read carefully, since the availability of autonomous 
cars needs to be an enabler for users, and high demand interfaces may limit perceived usefulness. Other than [19], where split positive and negative views between autonomous and non-autonomous cars were discovered, the authors are not aware of any other study that highlights this split of opinions on whether the in-between step of partial automation is useful for all.

\section{Conclusions}

This paper presented a survey with UK participants as a recruitment tool for later studies, and two focus groups with an inclusive user group of survey respondents that followed. The survey was novel in combining self-reported inclusive characteristics, driving habits, and acceptance of manual and autonomous driving. It demonstrated how even a small number of respondents can reveal clear attitude patterns towards manual driving, and achieve an acceptable spread of demographics. It also enabled later recruitment of users with distinct characteristics as part of an inclusive design methodology. The two focus groups, using survey participants of different inclusive characteristics, showed consensus in the topics of safety and usability of autonomous cars and HMIs, and the use of multimodal displays as a warning mechanism. They also revealed a split of opinions on whether partial autonomy is acceptable. However, different concerns between the focus groups were also discovered, with the younger participants seeing autonomy as an augmenter of already high capabilities, and the more inclusive group as an enabler for possible limitations. The implications of these findings for autonomous vehicle and HMI designers are discussed.

Acknowledgments. This work was funded by EPSRC and Jaguar-Land Rover, as part of the project Human Interaction: Designing Autonomy in Vehicles (HI:DAVe), Project Grant Number: EP/N011899/1.

\section{References}

1. Sage, A., Lienert, P.: Ford plans self-driving car for ride share fleets in 2021, Reuters, (2016).

2. Abbugao, M.: Driverless taxi firm eyes operations in 10 cities by 2020, Agence France Presse, (2016).

3. Hawkins, A.J.: Delphi and Mobileye are teaming up to build a self-driving system by 2019 , The Verge, (2016)

4. Sessa, C., Pietroni, F., Alessandrini, A., Stam, D., Delle Site, P., Holguin, C., Flament, M., Hoadley, S.: Results on the on-line DELPHI survey - CityMobil2. (2015).

5. Rödel, C., Stadler, S., Meschtscherjakov, A., Tscheligi, M.: Towards Autonomous Cars: The Effect of Autonomy Levels on Acceptance and User Experience. Automot. UI 2014. 1-8 (2014).

6. Schoettle, B., Sivak, M.: A survey of public opinion about autonomous and self-driving vehicles in the US, the UK, and Australia. (2014).

7. Schoettle, B., Sivak, M.: Public Opinion About Self-Driving Vehicles in China, India, Japan, The U.S., The U.K. and Australia. (2014).

8. Schoettle, B., Sivak, M.: Motorists' Preferences for Different Levels of Vehicle Automation. (2015).

9. Schoettle, B., Sivak, M.: Motorists' Preferences for Different Levels of Vehicle Automation: 2016. (2016).

10.Kyriakidis, M., Happee, R., De Winter, J.C.F.: Public opinion on automated driving: Results 
of an international questionnaire among 5000 respondents. Transp. Res. Part F Traffic Psychol. Behav. 32, 127-140 (2015).

11.Clark, B., Parkhurst, G., Ricci, M.: Understanding the socioeconomic adoption scenarios for autonomous vehicles: A literature review., Bristol (2016).

12.Clarkson, J., Coleman, R., Keates, S., Lebbon, C.: Inclusive design: Design for the whole population. Springer (2013).

13.Jeon, M., Politis, I., Shaldover, S.E., Sutter, C., Terken, J., Poppinga, B.: Towards Life-Long Mobility: Accessible Transportation with Automation. In: AutomotiveUI 2016 EA. ACM (2016).

14.Intelligent Mobility: Traveller Needs and UK Capability Study. (2015).

15.Clarkson, P.J., Huppert, F.A., Waller, S., Goodman-Deane, J., Langdon, P., Myerson, J., Nicolle, C.: Towards Better Design, 2010. (2012).

16.Meschtscherjakov, A., Tscheligi, M., Szostak, D., Ratan, R., McCall, R., Politis, I., Krome, S.: Experiencing Autonomous Vehicles: Crossing the Boundaries between a Drive and a Ride. In: CHI 2015 Extended Abstracts. pp. 2413-2416 (2015).

17.Meschtscherjakov, A., Tscheligi, M., Szostak, D., Krome, S., Pfleging, B., Ratan, R., Politis, I., Baltodano, S., Miller, D., Ju, W., Kun, A.: HCI and Autonomous Vehicles: Contextual Experience Informs Design. In: CHI 2016 Extended Abstracts. p. In Press. ACM Press (2016).

18.Silberg, G., Manassa, M., Everhart, K., Subramanian, D., Corley, M., Fraser, H., Sinha, V., Ready, A.W.: Self-Driving Cars: Are we Ready? (2013).

19.Bazilinskyy, P., Kyriakidis, M., De Winter, J.: An international crowdsourcing study into people's statements on fully automated driving. Procedia Manuf. 3, 2534-2542 (2015).

20.Ipsos Mori: Only 18 per cent of Britons believe driverless cars to be an important development for the car industry to focus on. (2014).

21.Jaxa-Chamiec, M., Fuller, R.: Understanding digital inclusion - A research summary, (2007).

22.Milner, H. ed: Digital Inclusion A discussion of the Evidence Base, (2007).

23.Bradley, M., Langdon, P., Clarkson, P.J.: Assessing the Inclusivity of Digital Interfaces - A Proposed Method. In: Antona, M. and Stephanidis, C. (eds.) Universal Access in HumanComputer Interaction. Access to Today's Technologies. pp. 25-33. Springer International Publishing (2015).

24.Körber, M., Bengler, K.J.: Potential Individual Differences Regarding Automation Effects in Automated Driving. Proc. XV Int. Conf. Hum. Comput. Interact. - Interacción '14. 1-7 (2014).

25.Souders, D., Charness, N.: Challenges of Older Drivers' Adoption of Advanced Driver Assistance Systems and Autonomous Vehicles. In: International Conference on Human Aspects of IT for the Aged Population. pp. 428-440. Springer (2016).

26.Kunur, M Langdon, P Bradley, M Bichard J-A Glazer, E Doran, F Clarkson, J Loeillet, J.: Creating Inclusive HMI Concepts for Future Cars Using Visual Scenario Storyboards Through Design Ethnography. In: International Conference on Universal Access in HumanComputer Interaction. pp. 139-149. Springer (2015).

27.Inclusive Design Toolkit, www.inclusivedesigntoolkit.com.

28.Politis, I., Brewster, S., Pollick, F.: Language-Based Multimodal Displays for the Handover of Control in Autonomous Cars. In: Automotive UI 2015. pp. 3-10. ACM Press (2015).

29.Mccall, R., McGee, F., Meschtscherjakov, A., Louveton, N., Engel, T.: Towards A Taxonomy of Autonomous Vehicle Handover Situations. Proc. 8th Int. Conf. Automot. User Interfaces Interact. Veh. Appl. (AutomotiveUI '16), Oct. 24-26, 2016, Ann Arbor, MI, USA. 193-200 (2016).

30.Flick, U.: An Introduction to Qualitative Research - Fourth Edition. SAGE (2009).

31.National Highway Traffic Safety Administration: Preliminary Statement of Policy Concerning Automated Vehicles. , Washington, DC (2013).

32.J3016, S.: Taxonomy and Definitions for Terms Related to Driving Automation Systems for On-Road Motor Vehicles. (2016). 